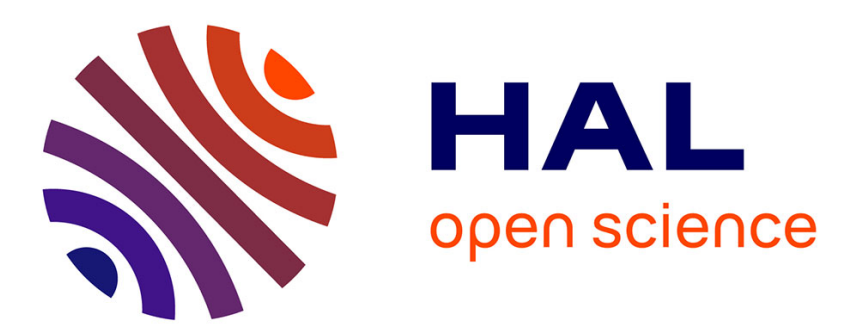

\title{
L'appropriation des espaces publics par les personnes sans-abri, entre contraintes et elaboration d'un "chez-soi"
}

Antonin Margier

\section{- To cite this version:}

Antonin Margier. L'appropriation des espaces publics par les personnes sans-abri, entre contraintes et elaboration d'un "chez-soi". Sociologia urbana e rurale, 2014, 104. halshs-01284643

\section{HAL Id: halshs-01284643 \\ https://shs.hal.science/halshs-01284643}

Submitted on 9 Mar 2016

HAL is a multi-disciplinary open access archive for the deposit and dissemination of scientific research documents, whether they are published or not. The documents may come from teaching and research institutions in France or abroad, or from public or private research centers.
L'archive ouverte pluridisciplinaire HAL, est destinée au dépôt et à la diffusion de documents scientifiques de niveau recherche, publiés ou non, émanant des établissements d'enseignement et de recherche français ou étrangers, des laboratoires publics ou privés. 


\section{L'APPROPRIATION DES ESPACES PUBLICS PAR LES PERSONNES SANS-ABRI, ENTRE CONTRAINTES ET ELABORATION D'UN «CHEZ-SOI»}

di Antonin Margier*

«Il n'y a pas d'être sans lieu d'être»

(Berque, 1997: 294).

L'itinérance et le sans-abrisme comptent certainement parmi les symboles les plus visibles de la pauvreté urbaine contemporaine. De plus en plus présent dans les espaces publics des métropoles, le phénomène interroge les chercheurs autant que les responsables politiques. Beaucoup visent alors à comprendre les phases de désaffiliation et les ressorts de l'exclusion menant à cette réalité (Paugam, 1991) tandis que d'autres analysent la mise à l'écart dont sont souvent victimes ces précaires, devenus «indésirables» dans le paysage mondialisé des villes modernes (Smith, 1996; Mitchell, 2003). Dans ces perspectives, les sans-abri seraient ballotés par des forces structurelles les dépassant et sur lesquelles ils n'auraient pas de prise. À travers cet article, nous souhaitons justement prendre en compte les pratiques mises en œuvre par ces individus dans leur occupation de la ville, et notamment en saisir les significations. En nous appuyant sur l'idée que ces personnes ne sont pas totalement démunies de moyens d'action (Girola, 2011; Parazelli, 1997; Zeneidi, 2008), nous visons à éclaircir les rapports qu'elles entretiennent à leurs espaces de vie et au quartier. Comment, à travers l'appropriation des espaces publics, ces personnes parviennent à donner un sens à leur propre expérience de vie dans la rue? Dans cette optique, nous portons notre attention sur les quartiers de la Goutte d'Or à Paris et du Village Shaugnessy à Montréal, dans lesquels la présence de personnes marginalisées ${ }^{1}$ suscite certains conflits avec les riverains. La persistance de

\footnotetext{
"Lab’Urba, Université de Paris-Est, antoninm@hotmail.fr

${ }^{1}$ Les situations des personnes sans-abri ou itinérantes étant multiples, nous utilisons le terme de personnes marginalisées afin de décrire ces individus. Cela permet notamment de décrire comment leur condition et leurs comportements les définissent comme des individus «out of place» (Cresswell, 1996) dans les espaces publics sous l'influence des normes inscrites dans l'espace urbain. Plutôt que d'évoquer les marginaux, le terme de «personnes marginalisées» permet en outre de s'abstraire de toute essentialisation de la situation de ces Sociologia urbana e rurale n. 104, 2014
} 
ces occupations laisse d'ailleurs à penser que des liens particuliers se tissent entre ces personnes et l'espace du quartier. Toutefois, pour en saisir la portée, il importe de revenir dans un premier temps sur le concept d'appropriation et d'expliciter notre posture théorique vis-à-vis de l'espace géographique. Par la suite, nous présenterons nos résultats en nous basant sur des entrevues de terrain.

\section{Réflexions sur l'appropriation de l'espace géographique}

\subsection{L'importance des discontinuités dans l'espace géographique}

S'attacher à comprendre l'importance prise par le quartier pour les personnes sans-abri, et en particulier par les espaces publics du quartier, implique de clarifier notre posture épistémologique. Car l'espace n'est ni une substance neutre sur laquelle seraient projetés des rapports sociaux ni le résultat des seules interactions sociales mais résulte davantage de l'interactivité entre la subjectivité de nos mondes intérieurs et la matérialité du monde extérieur. Lier une approche phénoménologique aux enjeux socio-politiques pour penser l'espace nous semble alors nécessaire afin d'éclairer les logiques de son investissement et le sens des pratiques sociospatiales. Or, la géographie structurale, en inscrivant la discontinuité et l'hétérogénéité au cœur de l'interprétation de l'espace géographique, permet d'intégrer la conception topologique de l'espace (Desmarais, 1992; Hubert, 1993) à la compréhension des pratiques sociales de l'espace. Revenons alors dans un premier temps sur les points principaux de cette approche.

Plusieurs auteurs se sont attachés à montrer l'importance fondamentale de l'espace dans l'existence humaine, l'être humain étant «un être géographique» (Berque, 2009: 10). Or, loin de ne constituer qu'un contenant homogène, l'espace est au contraire hétérogène et anisotrope (Desmarais et Ritchot, 2000). Au cours des mouvements de l'être humain, sa perception de l'espace évolue et les «qualités d'espace peuvent être associées à des lieux déterminés, c'est-à-dire à des positions sur la Terre» (Hubert, 1993: 31). La position n'est donc plus une simple mesure géométrique mais «un véritable phénomène» (Hubert, 1993: 39). Loin de n'être que la localisation abstraite d'un lieu, la position est le résultat de phénomènes de mobilité et de représentations, et «intervient dans l'existence de l'être géographique» (Hubert,

personnes et soulève également les rapports de pouvoir à l'œuvre dans le processus de marginalisation. 
L'appropriation des espaces publics par les persone sans-abris

1993: 39), au point que «la géographie structurale considère la représentation topologique de l'espace comme étant la forme ontologique de l'espace géographique» (Parazelli, 1997: 154). Les tenants de la géographie structurale proposent alors de penser l'espace géographique comme: «[...] un système de discontinuités qualitatives qui se situe à l'interface d'un niveau observable de surface, celui des formes architecturales concrètes, et d'un niveau profond non observable, comprenant des significations symboliques dont l'essence est non spatiale» (Desmarais, 1992: 254). Associées à des positions, ces significations symboliques joueraient un rôle fondamental dans la structuration de l'espace puisqu'elles déclencheraient, en fonction de leur attractivité ou de leur répulsivité, des désirs d'appropriation chez les individus $^{2}$, instaurant alors des rapports de force entre ceux qui possèdent le contrôle de leur mobilité et ceux qui sont contraints par la mobilité des premiers (Desmarais et Ritchot, 2000). Parazelli (1997) évoque ainsi l'image de l'échiquier pour décrire cette structuration abstraite de l'organisation et de l'investissement de l'espace géographique, et l'importance stratégique que revêtent certains emplacements par rapport à d'autres. Des emplacements vides peuvent en effet s'avérer stratégiques à s'approprier, notamment par leurs positions et leurs relations à d'autres emplacements.

\subsection{L'appropriation, un processus d'identification}

L'attractivité d'une position induit donc des processus d'appropriation, impliquant la projection de soi ou du collectif dans celle-ci. Les valeurs spatialisées dans l'espace par l'appropriation (Ripoll et Veshambre, 2006) constitueraient alors des «références utilisées par un et/ou des acteurs (...) pour se définir en se distinguant des autres acteurs» (Lussault, 2007: 93). Cette conceptualisation témoigne de l'importance des logiques de distinction et de reconnaissance qui accompagnent la mise en œuvre des stratégies d'appropriation. En évoquant l'appropriation de la maison, Serfaty-Garzon

\footnotetext{
${ }^{2}$ La notion de prise telle que l'a interprété Augustin Berque renvoie également à l'importance ontologique de l'appréhension subjective de l'espace: «Un milieu se manifeste en effet comme un ensemble de prises avec lesquelles nous sommes en prise: des ressources et des contraintes, des risques et des agréments que la réalité comporte dans la mesure où elle nous comporte aussi, et où nous les prenons comme tels; qui ne sont ni vraiment des objets physiques, puisqu'ils n'existent qu'avec nous, ni vraiment des phénomènes subjectifs, puisque leur vérité s'ancre dans les choses. Bref, ce sont des réalités mésologiques: ni l'ensoi de la physique, ni le pour-soi de la psychologie, mais l'avec-soi d'un potentiel qui se réalise dans la relation d'une société à l'espace et à la nature» (Berque, 1990: 103).
} 
évoque clairement ce processus, «le tissage de significations entre l'habitant et sa maison issu des pratiques n'est pas seulement de l'ordre du marquage ou de la personnalisation, mais de l'identification et de l'inscription d'un mode d'être» (Serfaty-Garzon, 2003: 29-30). Le processus d'appropriation intègre ainsi l'appartenance sociale à un groupe, à un ensemble de valeurs associées à ce dernier:

D'une part, le marquage, par la disposition des objets ou les interventions sur l'espace habité, est l'aspect matériel le plus important de l'appropriation. D'autre part, ces qualités de lieu personnel ne sauraient exister sans l'existence d'un "nous" qui en cautionne la légitimité, sans les valeurs qui leur sont attachées, c'est-à-dire sans l'existence d'un «modèle culturel» qui en inspire et fonde l'organisation (Serfaty-Garzon, 2003: 29).

Ainsi, si certaines significations symboliques associées à une position attirent certains individus parce qu'ils s'y reconnaissent et y voient un potentiel d'identification, l'investissement par des individus de valeurs dans une position participe également à l'élaboration d'un espace de reconnaissance. Ces propos soulèvent donc l'importance avec laquelle l'appropriation d'un lieu peut participer à l'édification d'un «chez-soi» identificatoire. Favorisant des processus de reconnaissance de soi, l'appropriation d'une position permet ainsi de créer un espace protecteur, «même sans amplificateurs architecturaux massifs, tout groupe se désignant par un "Nous" sait se mettre à couvert dans une figure sensible» (Sloterdijk, 2011: 176). Ces réflexions laissent d'ailleurs entrevoir le fait que le «chezsoi» n'est pas nécessairement lié à l'habitat en lui-même, mais qu'il peut également se constituer dans les espaces publics, et à travers leur appropriation en particulier. Car «anthropologiquement, l'homme a besoin d'espace, mais plus encore d'un lieu» (Moles et Rohmer, 1978) et «l'individu essaie (...) toujours, quelles que soient les conditions particulières dans lesquelles il se trouve, de sauvegarder un espace personnel» (Fischer, 1981: 94).

De fait, même les pratiques ordinaires de ceux qui n'ont pas d'abri peuvent favoriser l'appropriation de certains lieux, à travers l'occupation, le marquage, et en particulier par les relations sociales qui s'y déroulent. La réciprocité des relations (Parazelli, 1997) et la coexistence deviennent alors fondamentales dans le sens attribué aux différentes positions, elle [la coexistence] est «ce qui rend l'espace possible» (Sloterdijk, 2005: 271). Les relations sociales sur lesquelles se manifeste l'appropriation favoriseraient alors la constitution de sphères et la différenciation topologique de l'espace: 
L'appropriation des espaces publics par les persone sans-abris

ceux qui sont rassemblés dans l'espace psycho-et sociosphérique constituent euxmêmes l'espace par la force de leur coexistence: ils sont imbriqués les uns dans les autres et constituent, sur le mode de l'abri que l'on s'offre l'un à l'autre et de l'évocation réciproque, un lieu psychosocial d'un type spécifique (Sloterdijk, 2005: 271-272).

Comme Parazelli l'a montré à propos des jeunes de rue, l'appropriation de certains espaces publics leur permettrait de poursuivre un processus identitaire, souvent perturbé au cours de l'enfance à cause de rapports de domination, de violence, et généralement de la non-reconnaissance à laquelle ils ont fait face. Dans ces lieux, ils créeraient notamment un espace de socialisation, dans lequel trouver de la reconnaissance, et leur permettant d'édifier un processus d'identification:

L'espace et son appréhension symbolique représentent un point d'appui psychosocial fondamental de la structuration identitaire, car c'est par l'appropriation de lieux (si elle perdure dans le temps) que l'individu peut accomplir et stabiliser un processus d'identification, l'espace représentant le foyer de toutes les expériences possibles (Parazelli, 2002: 141).

Ces réflexions soulignent donc l'importance de l'appropriation dans le processus de constitution d'un chez-soi, d'un espace domestique. Or, comme le signale Hoyaux, «[...] l'espace domestique ne doit pas être conçu uniquement comme un contenant délimité par les murs d'un appartement ou la clôture d'une maison d'habitation principale, mais aussi, et peut être surtout, comme un espace contenu où la projection de l'être, en chair, construit un monde où sa sécurité ontologique est maximum» (Hoyaux, 2004: 33). Si l'on s'appuie sur l'apport de Giddens, la sécurité ontologique renvoie à « la confiance de la plupart des êtres humains dans la continuité de leur propre identité et dans la constance des environnements d'action sociaux et matériels» (Giddens, 1994: 98). Dans cette mesure, la constitution d'une sécurité ontologique est clairement liée à l'appropriation de lieux à travers lesquels se reconnaître et dans lesquelles la confiance puisse être maximale. Mais, ce que soulève également Giddens, c'est l'importance des routines dans l'élaboration de cette sécurité, «l'aspect prévisible des routines (apparemment) mineures de la vie quotidienne est profondément liée au sentiment de sécurité psychologique» (Giddens: 104).

Cette conceptualisation de l'appropriation de l'espace géographique permet donc d'interroger l'usage des espaces publics par les personnes sans-abri et marginalisées. Leurs pratiques sociospatiales peuvent en effet s'avérer porteuses de significations que les chercheurs et les experts ont, de 
prime abord, tendance à négliger. Pour des personnes ne possédant pas d'espace privé et intime, l'appropriation des espaces publics peut participer à combler ce manque, et le quartier (et ses espaces publics en particulier) s'avérer «une «ressource» relationnelle et identitaire» (Grafmeyer, 2007: 30). Les résultats empiriques qui suivent visent alors à illustrer ces propos.

\section{Les espaces publics du Village Shaugnessy et de la Goutte d'Or: des espaces de vie}

Situés respectivement dans le 18è arrondissement de Paris et à l'ouest du centre-ville de Montréal, les quartiers de la Goutte d'Or et du Village Shaugnessy font l'objet depuis plusieurs années d'un processus de gentrification (Bacqué et Fijalkow, 2006; Corral, 1986), en parallèle duquel émergent de nouvelles exigences résidentielles. Or, chacun de ces quartiers connaît une occupation de ses espaces publics par des personnes en situation de précarité, sans-abri, toxicomanes et prostituées, dont la présence et la visibilité suscitent des conflits et certaines mobilisations de riverains (Margier, 2013). Au-delà de ces similitudes, c'est la persistance du phénomène qui nous a incité à élaborer une comparaison entre ces deux quartiers. En effet, tandis que le square Cabot et certains secteurs du Village Shaugnessy sont associés à l'itinérance autochtone depuis plus d'une quinzaine d'années, la Goutte d'Or constitue un quartier dans lequel se concentrent depuis plus de vingt ans des enjeux liés à la précarité mais également à la toxicomanie ou la prostitution. Si cette occupation des espaces publics par des populations marginalisées semble considérée comme une nuisance au confort résidentiel des riverains, il importe également de comprendre le sens de cette présence dans le quartier ainsi que les mécanismes à travers lesquels ces individus s'y inscrivent comme habitants ${ }^{3}$.

\footnotetext{
${ }^{3} \mathrm{Au}$ niveau méthodologique, nous nous appuyons sur une douzaine d'entrevues réalisées lors de notre recherche doctorale avec des personnes marginalisées occupant les espaces publics dans les deux quartiers. Pour y parvenir, nous sommes tout d'abord entrés en contact avec des organismes communautaires et des travailleurs sociaux, par l'entremise desquels nous avons ensuite été introduits parmi les personnes marginalisées. L'ensemble des personnes interrogées sont sans-abri et itinérantes (hormis 3 répondants sortis récemment de la rue mais qui continuent à fréquenter ces quartiers), et bien que la majorité dorment dans des refuges ou dans des squats, elles investissent quotidiennement les espaces publics étudiés. Les entrevues ont été retranscrites et analysées, laissant apparaître des convergences et des divergences entre les deux terrains mais surtout des tendances générales à propos des usages des espaces publics, relatives à l'ensemble des répondants. Pour des raisons évidentes de manque de place, nous avons, dans le cadre de cet article, dû restreindre
} 
L'appropriation des espaces publics par les persone sans-abris

\subsection{Des espaces de sociabilités}

Dès lors que l'on s'intéresse à l'usage des espaces publics des deux quartiers, les rencontres et les sociabilités s'avèrent d'une importance majeure. Dans le Village Shaugnessy, le square Cabot - l'un des parcs centraux -, souvent occupé par les personnes marginalisées, constitue en effet un lieu de rencontre dans lequel construire du lien social. Différentes pratiques accompagnent ce processus, il peut s'agir d'activités illicites (consommation de drogues ou d'alcool) ou simplement de repas partagés le soir et de discussions collectives autour des tables disposées dans le square. Les interactions sociales se stabilisent ainsi en ce lieu, «it's like the only place for them, because that's where they all meet friends» (Personne Marginalisée, PM2). De fait, les projections familialistes (Parazelli, 2002) parcourent les discours des répondants pour illustrer les liens sociaux et les solidarités qui se manifestent dans ces pratiques d'appropriation, «it's like a family there, (...). It's like if somenone is missing for a couple days not here, we start to wonder where they are, what happen to them...» (PM1). Tout un processus de reconnaissance est ainsi à l'œuvre dans ces pratiques d'appropriation L'un des répondants insiste notamment sur le fait de connaître une grande partie des occupants des espaces publics, mais d'y être également reconnu, «I used to go there the first time when I came here... I know maybe all of them, the people who live in the street (...), lots of friends. Men and women. And everybody knows me» (PM5). L'usage et l'occupation de cet espace public permettent donc à ces personnes de développer une appartenance sociale et une identification spatiale. Ainsi, cette familiarité au lieu et la reconnaissance qui s'y manifeste renforcent l'attractivité de cette position pour beaucoup de ces personnes marginalisées, «they're other places but that's just the main place to go. And everyone knows everybody there...» (PM1). La réciprocité des relations (Parazelli, 1997) joue donc un rôle important dans la valorisation symbolique et l'usage du square.

Dans les espaces publics de la Goutte d'Or, ce processus se fait également jour. Bien que la présence de vendeurs de stupéfiants soit évoqués par plusieurs répondants comme l'une des raisons de leur présence dans le quartier, «il y a des amis et la facilité d'obtenir la drogue, le produit (...). Oui, Il y a beaucoup de choses qui m'intéressent [dans le quartier]. Déjà, la priorité, ça c'est clair, c'est la drogue» (PM10). La présence de stupéfiants

les exemples, et avons donc sélectionné des extraits d'entrevues qui soient le plus représentatifs de ces résultats. 
et la connaissance du milieu permettent également à certaines personnes marginalisées de faire quelques trafics, «tu peux même [dans le quartier] faire ton argent tranquillement, mais il faut être prudent» (PM10). Les stratégies de débrouille liées à la connaissance du quartier leur permettent ainsi de gagner quelque monnaie lorsqu'elles sont dans le besoin:

[...] des fois, quand j'ai pas d'argent, tu sais... dans le métro, il y a des petits trafics, de $\operatorname{sub}^{4} \ldots$ des fois je suis fauché, je prends deux euros, j'achète une plaquette, je revends et ça me fait un peu de sous pour m'acheter de quoi tenir, des cigarettes, et tout, etc... (PM9).

Les opportunités de survie et de débrouille qui se présentent dans le quartier à qui le connaît participent donc de son attractivité sur certaines personnes marginalisées. Cependant, au delà de ces aspects utilitaristes de l'occupation des espaces publics du quartier, nous constatons qu'une importance particulière est accordée aux relations sociales qui y sont développées et qui leur permet de s'y sentir appartenir.

La majeure partie des répondants étant d'origine immigrée ou des territoires d'outre-mer, la présence de personnes aux origines communes est alors considérée comme un atout pour s'en sortir. D'origine africaine et arrivé en 2011 à Paris de province, l'un des répondants est venu spécifiquement à la Goutte d'Or dès son passage à la rue, sur les conseils de personnes rencontrées. Il a ainsi pu rencontrer des individus dans sa situation et partager ses difficultés, notamment avec des Africains, avec qui, affirmet-il, les relations de soutien et d'aide étaient plus fortes, «il y a beaucoup d'endroits où on peut t'aider. Tu peux rencontrer beaucoup d'Africains, les gens ils ont pitié de toi, si t'a pas de moyens, tu peux demander, on te donne un euro ou deux euros» (PM7). Beaucoup d'Africains en situation de marginalité trouvent dans ce lieu, symbole d'une «centralité immigrée» (Toubon et Messamah, 1990), des liens sociaux grâce auxquels se faire une place et obtenir une certaine reconnaissance. Les sociabilités offertes par la fréquentation du quartier semblent alors particulièrement importantes:

[...] parce qu'ici, il y a tous les Africains, il y a beaucoup d'Africains qui passent ici, c'est pour ça, nous tous, on... tu vois pas, si tu restes là [dans le square Léon, au centre du quartier] longtemps jusqu'à la fermeture, toujours il y a des gens qui

\footnotetext{
${ }^{4}$ Le Subutex est un substitut aux opiacés utilisé dans le contexte des cures de désintoxication mais également consommé par de nombreux toxicomanes. Il se développe notamment dans le quartier un trafic de ce médicament.
} 
L'appropriation des espaces publics par les persone sans-abris

viennent, toujours. C'est pour ça que moi, je ne fréquente pas un autre lieu qu'ici... (PM7)

Les relations sont donc importantes dans l'occupation des espaces publics, et la présence d'individus avec qui tisser des liens sociaux a ainsi permis à ce répondant d'appréhender l'espace de la rue, de connaître les solutions pour survivre et satisfaire ses besoins vitaux, «j’ai trouvé beaucoup de gens que je connais ici, on a discuté, comment ça se passe» (PM7). Ces relations lui ont permis de profiter de l'aide de plusieurs individus, de pouvoir se réchauffer parfois chez certains tout en développant un réseau de sociabilités, «il y a beaucoup d'amis» (PM7). Sans domicile fixe, il a ainsi pu s'affirmer comme habitant du quartier et développer un sentiment d'appartenance particulièrement fort, qui lui donne aujourd'hui l'envie d'y rester:

C'est pour ça que toujours, je suis là, c'est pour ça que tous les jours... Même parfois, je pars jusqu'à Beauvais [en Picardie] dormir, un jour, mais je ne vais pas rester plus d'une journée, pourtant j'ai des amis la bas qui me disent de rester dormir, etc., mais je dis non parce que là-bas, c'est la merde. Moi tous les jours, il faut que je vienne dans le $18 \mathrm{e}$, si je ne suis pas dans le parc, je suis à $\mathrm{EGO}^{5}$, si je ne suis pas à EGO, je suis à la boutique, le soir...(...). Après on n'a pas d'endroit où prendre le café, on n'a pas d'endroit où aller, on vient à côté de franprix, si t'as des bières tu bois, si t'en as pas, si t'as envie de dormir, tu dors, si t'as envie de discuter, il y a des chaises et des matelas, des fois on discute jusqu'au matin, c'est comme ça, ça passe (PM7).

L'existence d'un réseau social explique ainsi clairement sa présence dans le quartier et l'occupation de ces espaces publics. Fréquentant parfois certains lieux extérieurs, une autre personne justifie d'ailleurs sa préférence pour ce quartier, du fait de la présence de connaissances qui participe à son bien-être:

I: C'est ici tes endroits préférés, t'en fréquentes d'autres?

R: Oui, mais beaucoup moins. C'est aussi une question d'habitudes, on se connaît, on est à l'aise...(PM8).

Par ailleurs, même l'un des répondants (PM11) selon qui la recherche de drogue primerait sur toute sociabilité et qui parle d'un «chacun pour soi», reconnaît tout de même la «grande famille» que constitue le quartier.

\footnotetext{
${ }^{5}$ Espoir Goutte d'Or est une association de soutien aux personnes toxicomanes localisée dans le quartier de la Goutte d'Or.
} 
Loin d'être seulement guidée par des logiques utilitaristes, l'appropriation des espaces publics répond dans les deux cas aux stratégies personnelles et collectives d'occupation de l'espace et le marquage correspondrait comme le suggère Zeneidi-Henry à des «besoins de survie, de contrôle d'un espace personnel, de reconnaissance, de sociabilité et d'identification» (ZeneidiHenry, 2008: 271). Cependant, au-delà de l'importance relationnelle et sociale de l'occupation de ces lieux, cette reconnaissance se manifeste également dans la constitution des espaces publics comme un «chez-soi».

\subsection{Des espaces publics instaurés comme «chez-soi»}

En effet, dans le Village Shaugnessy, le square Cabot semble être mobilisé comme un lieu protecteur et rassurant, dans lequel plusieurs répondants affirment se retrouver le soir, après des journées dans le reste de la ville, pour des activités plus sociales et festives. À travers les relations qui prennent forme dans son occupation sociale, c'est la dimension protectrice de cette position qui se constitue. Le square joue alors clairement le rôle d'un «chez-soi» familier dans lequel se relâchent certains réflexes défensifs, de vigueur dans certains autres lieux comme le parc Viger:

I just want to be here, often if we can't go there, we go there on Viger, Viger Square, I don't like it on there because it's dangerous, I don't like... (...) I have my family there [in Cabot Square] so I'm not in danger, I feel more danger when i'm alone (PM1).

L'occupation des espaces publics du quartier et du square Cabot en particulier par ces personnes traduit alors un attachement fort aux lieux et renvoie à une certaine forme d'appartenance, «this space is basically their home, during the day I mean...» (PM3). Cette appartenance est d'autant plus manifeste pour les itinérants autochtones et Inuits, pour qui ce square est clairement reconnu comme un point de rencontre, «it's like a reuniting place for Inuit people these days (...) it's a uniting place, when they're separate in the world they come back there to meet up again, to meet other people» (PM3). Ainsi, il est clairement apparu au fil des entrevues que l'occupation du parc et des espaces publics aux alentours relevait d'une forme d'appropriation participant à la constitution d'une appartenance au quartier. Comme nous l'a confié une personne qui alterne depuis plusieurs années l'occupation de logements sociaux et la vie dans la rue, sa connaissance intime des lieux du quartier renvoie à un «chez-soi», «ça fait 30 ans 
que je viens ici, c'est comme chez moi» (PM4). Ces références récurrentes $\mathrm{au}$ «chez-soi» et à l'appartenance témoignent notamment du fait que ces personnes habitent le quartier.

Comme nous le constatons également à la Goutte d'Or, la connaissance et l'habitude des lieux participent à cette constitution d'un «chez-soi», «je connais bien (...) le quartier, par rapport à un certain nombre qui vivent ici, qui dorment ici H24, moi je connais des trucs qu'eux-mêmes ne connaissent même pas» (PM10). Comme l'un des répondants, sans-domicile et toxicomane, qui dort dans un foyer $\mathrm{du} 1^{\mathrm{er}}$ arrondissement depuis bientôt deux ans, mais qui revient tous les jours à la Goutte d'Or, «j'ai mes repères, je suis à l'aise, je suis chez moi ici» (PM8). Bien que la plupart de ces répondants dorment ailleurs, dans des refuges ou dans des squats, ils fréquentent quotidiennement le quartier, et s'y identifient:

Ah oui, les gens ils habitent plus le 18e que leur propre quartier. La plupart ne fréquentent pas leur quartier, ils ont [un endroit] où ils dorment, où ils squattent, mais ils sont plus d'ici que ... Ça, c'est un fait, c'est comme moi, je me sens plus d'ici... (PM8).

Ces propos sont confirmés par un autre usager de drogues, toxicomane depuis 20 ans et qui ne quitte guère le quartier que pour aller dormir, «franchement, pour moi, je me sens vraiment ... comme si j'habitais dans le $18 \mathrm{e}$ parce que je connais toutes les petites rues (...). Parce que je vis plus ici, je suis plus dans le 18 que dans ma propre banlieue» (PM10). Il se développe ainsi chez l'essentiel des répondants, au fil de leurs pratiques de ces espaces publics, un sentiment d'appartenance sociale et spatiale au quartier de la Goutte d'Or. Au-delà du sentiment d'appartenance au quartier que l'on peut observer chez les personnes marginalisées, c'est l'appréciation du lieu qui se manifeste dans leur discours, «même une semaine en évitant de venir dans le quartier, ça vous manque, tout de suite» (PM10). La dimension sensible des souvenirs ainsi que les rapports affectifs et symboliques aux lieux jouent alors un rôle important dans cet attachement au quartier:

[...] des bons souvenirs comme des mauvais souvenirs, il y a tout quoi, franchement j'aime bien ce quartier (...). Mais sinon, le quartier franchement, moi je l'aime, je l'aime bien. Pourtant, j'y suis pas né ici, mais je l'aime bien, je me suis vraiment adapté, franchement, ouais (PM10).

L'habitude des lieux participe donc de leur appropriation, tout en renforçant le sentiment d'être du quartier et construisant le cadre social auquel ces personnes se sentent appartenir. 
Cette dynamique sociale à l'œuvre parmi les personnes marginalisées témoigne de la valeur accordée au quartier et à ses espaces publics à travers l'usage desquels il leur devient possible de développer des stratégies de survie et de satisfaire des sociabilités. Les pratiques et les discours des personnes marginalisées témoignent également de la familiarité qu'elles entretiennent avec le quartier et de leur sentiment de l'habiter. Sans être des "citoyens" du quartier au «sens pleinement politique du terme, ils en sont les habitants, parce qu'ils y ont un emplacement dans lequel ils vivent, qui les identifie comme tels et les inscrit dans une relative familiarité du lieu» (Lazzarotti, 2006: 108). Cela témoigne notamment de la sécurité ontologique que construisent ces personnes dans l'appropriation de cette position. L'interconnaissance et le regroupement qui lui est lié participent ainsi de la constitution d'une protection, d'un sentiment de confiance en ce lieu (Giddens, 1994). Cela leur permet notamment de reconnaître les personnes qui n'y ont pas leurs habitudes, «because everybody knows everybody, we can recognise them, you know» (PM5). À travers l'investissement de cet espace dans lequel se joue le développement d'une familiarité et la projection de valeurs symboliques, il apparaît une démarcation topologique avec le reste de l'espace urbain, et la constitution d'un "chez-soi" protecteur pour ces personnes marginalisées, dans lequel se reconnaître.

\subsection{De l'importance des liens sociaux développés dans les espaces publics}

Il se développe ainsi chez l'essentiel des répondants, au fil de leurs pratiques de ces espaces publics, un sentiment d'appartenance sociale et spatiale aux quartiers concernés. À travers le fait d'être connu dans le quartier, de connaître du monde, de faire partie d'un groupe, la plupart des personnes interrogées trouvent un sentiment de reconnaissance. L'importance des liens sociaux développés dans la rue et les espaces publics se manifeste notamment dans la difficulté qu'éprouvent certains à les quitter pour intégrer un logement ou mettre en œuvre un processus de réinsertion.

Dans le Village Shaugnessy, plusieurs répondants, récemment logés ou inscrits dans une dynamique de scolarisation, ont ainsi évoqué leur prise de distance avec le square, avec ces lieux qui ne sont plus considérés comme protecteurs et seraient même devenus dangereux, «they want to stay there to do alcoohol or drugs (...). It wasn't like that in 2006 (...). I wouldn't live there, in Atwater area, no... I prefer stay away from this place, focus on my school» (PM3). Cela semble répondre à une volonté de se démarquer d'une population souvent stigmatisée et de marquer plus nettement sa sortie de 
rue et son intégration. Pour autant, cette personne continue régulièrement à aller dans le square pour voir des amis, qui continuent d'occuper l'endroit, «i don't see them everyday, but once or twice a week I try to go see them. Sometimes, they're there, sometimes they're not there (...). Atwater park, that's where I usually meet my friends» (PM3). De la même manière, une répondante, depuis 20 ans dans la rue et ayant beaucoup fréquenté le square Cabot, est inscrite dans un processus de réinsertion, grâce à un programme de scolarisation et à un traitement contre ses addictions. Passant d'appartements en refuges, elle juge le square Cabot de plus en plus dangereux au fil des ans, et dit l'éviter depuis qu'elle a une fille. Elle continue cependant à le fréquenter, car certains de ses amis restent présents dans le parc, «my friends are there, I go there sometimes, I go see my friend, but when she's too drunk, i just walk away, you know like ... (...) because she's on crack, pff ...»(PM2). Pourtant, malgré les récriminations qu'elle attribue au square, elle y retourne régulièrement pour les sociabilités et les amitiés qu'elle y a auparavant développées et s'y estime encore bienvenue, «because i see my friends there, my old friends, one of my old best friends is hanging around 24 hours, seven days a week over there» (PM2). Cet attachement aux relations sociales développées et au lieu dans lequel elles se déroulent peut parfois rendre difficiles les conditions du passage dans un logement. Ainsi, l'une des répondantes a évoqué sa difficulté à s'adapter à la vie en appartement. Du fait qu'elle était enceinte, les services sociaux ont récemment réussi à lui trouver un logement à l'est du centre-ville pour élever son enfant à venir. Or, cet accès au logement n'est pas vécu comme un soulagement. Au contraire, elle ne s'y sent pas à l'aise et regrette régulièrement la vie dans la rue, ce qui l'amène à continuer de fréquenter quotidiennement le square et ses amis qui y sont encore, «I still hang up with them, I seat there with all them... I kind of miss it» (PM1). Quant à savoir si elle se sent plus appartenir au quartier dans lequel est situé son logement ou au square, sa réponse est sans ambages, «I feel more from Cabot square, I'll always gonna be there, even... I don't know. That'll always be a part of... always be a part of our place. It's like home» (PM1).

L'histoire de l'une des personnes interrogées dans le quartier de la Goutte d'Or, âgée de 60 ans et ayant connu l'évolution du quartier qu'elle fréquente depuis pratiquement 40 ans, illustre également cette dynamique. Suite à une rupture, lors de la fuite de sa femme et de sa fille, cet individu a perdu son emploi puis son appartement, se retrouvant alors à la rue. Multipliant les combines pour se loger, entre appartements d'amis, refuges pour sans-abri, camion, etc., il a connu une instabilité particulièrement forte avec de nombreux et longs passages dans la rue: 
On était propriétaire à ce moment... mais moi, je suis retombé un peu là dedans...(...). J'avais mon camion, je dormais dedans... je connaissais toutes les filles du boulevard...j'étais dedans, j'avais pas d'argent, j'avais tout bazardé... je faisais le taxipute qu'on appelle et quand elles avaient besoin, je les amenais, elles me faisaient fumer... après, j'ai trouvé où squatter, mais je faisais le 115 de temps en temps pour me reposer... une fois que je discutais avec le 115, ils m'ont trouvé un foyer, un accueil, c'est l'armée du salut... j'étais donc là, je mangeais, je dormais, mais le matin, il fallait sortir...(PM9).

Ce passage de squats temporaires en refuges a récemment pris fin avec son accès à un logement transitoire, qui s'inscrit dans une optique de réinsertion et d'une possible embauche, «ils ont fait une demande au palais du peuple dans le 13e, le CCAS, t'as ta chambre, tu payes 120 euros, t'es pas emmerdé, t'as ta petite chambre, t'es tranquille. Je suis là, ça fait deux ans maintenant...» (PM9). Il possède ainsi un abri dans lequel dormir et se reposer dans le $13^{\mathrm{e}}$ arrondissement, géographiquement situé à l'opposé du quartier de la Goutte d'Or. Pourtant, malgré cette distance, il y revient encore très régulièrement, notamment car ses amis et ses connaissances y vivent, tandis qu'il lui est plus difficile de créer du lien social à proximité de son logement, «là-bas, il n’y a personne, c'est youpin (...). Quand je veux voir du monde, je viens ici... je discute un peu» (PM9). Au vu de son âge et de son expérience, il s'estime respecté dans le quartier et connu de l'ensemble des autres personnes marginalisées:

Là je viens régulièrement, j'avais arrêté. J'ai des copains, quand je n'ai rien à faire, alors je viens parler avec eux (...) du coup, les heures creuses, je viens les passer là, à l'association [EGO], je viens discuter, je parle, je suis celui qui... je les connais tous, mais je ne suis pas ...je suis à part, parce que c'est une jeune génération qui est là, les anciens ont disparu, une grande partie est morte, hommes, femmes...Voilà quoi...je les regarde, je me dis, je me pose des questions, comment ils font...il y a en a qui vont voler après ils vont faire la manche pour acheter un sandwich, ils ne pensent pas à après...à prévoir (...). Ben oui, je les connais tous, ils me connaissent, même à EGO, je les connais tous. Et ils ont un certain respect pour moi...Parce qu'ils m'ont vu dans ma déchirure, ils ont vu quand même que j'ai repris, parce que j'ai des gosses (PM9).

Ainsi, cette personne revient régulièrement dans le quartier au vu des amitiés et du lien social qu'elle y trouve ainsi que de la reconnaissance de soi qui s'y manifeste. Malgré le fait d'avoir un logement dans un autre quartier, l'appartenance sociale de cet individu renvoie clairement aux personnes marginalisées occupant les espaces publics de la Goutte d'Or. 
L'appropriation des espaces publics par les persone sans-abris

\section{Conclusion}

Nous voyons ainsi comment, à travers les sociabilités qui se développent dans les espaces publics, ces individus parviennent à créer un cadre social dans lequel se reconnaître mais également à développer un sentiment d'appartenance au quartier. Loin de constituer une «non-expérience vide de social et vide de sens» (Girola, 2011: 15), cette vie précaire dans la rue représente plutôt une véritable expérience dans laquelle tout un travail d'affirmation de soi est à l'œuvre. Dans l'appropriation des espaces publics, les personnes marginalisées, démunies et précarisées, parviennent à les instituer comme des espaces de vie dans lesquels trouver une reconnaissance et prendre place dans la ville. En les instaurant comme un «chez-soi» protecteur, ils comblent notamment l'absence de sécurité ontologique liée à l'absence d'espaces privés et intimes. Loin d'être déconnectées de toute vie sociale et d'intérêts comme cela est souvent évoqué, les personnes marginalisées interrogées révèlent au contraire l'importance de leurs relations sociales développées dans la rue mais également des rapports aux espaces publics et au quartier. Investis de valeurs particulières, les espaces publics des quartiers étudiés constituent alors des positions attractives, grâce à l'appropriation desquelles ces individus parviennent à habiter. Loin d'être dépourvues de significations, les pratiques sociospatiales des sans-abri devraient donc être davantage prises en compte, tant dans les stratégies de rénovation urbaine que dans l'élaboration des politiques de lutte contre le sans-abrisme.

\section{Rèfèrences bibliographiques}

Bacqué, M-H, Fijalkow Y. (2006). En attendant sa gentrification. Sociétés contemporaines, 63. doi: $10.3917 /$ soco.063.0063

Berque A. (1990). Médiance. De milieux en paysages. Montpellier: Éditions Reclus.

Berque A. (1997). Basho, chôra, Tjukurrpa, ou le poème du monde. L'espace Géographique, 4. doi: 10.3406/spgeo.1997.1094

Berque A. (2009). Écoumène. Introduction à l'étude des milieux humains. Paris: Belin.

Corral I. (1986). Inner city gentrification: the case of Shaugnessy Village, Montreal. Supervised Research Project. School of Urban Planning, McGill University.

Creswell T. (1996). In place, Out of place: geography, ideology, and transgression. London: University of Minnesota Press.

Desmarais G. (1992). Des prémisses de la théorie de la forme urbaine au parcours morphogénétique de l'établissement humain. Cahiers de Géographie du Québec, 98. doi: 10.7202/022268ar

Desmarais G., Ritchot G. (2000). La géographie structurale. Paris: L'Harmattan.

Fischer G-N. (1981). La psychosociologie de l'espace. Paris: Presses Universitaires de France. 
Giddens A. (1994). Les conséquences de la modernité. Paris: L'Harmattan.

Girola C. (2011). Vivre sans abri. Paris: Éditions Rue d'Ulm.

Grafmeyer Y. (2007). Le quartier des sociologues. In Authier J-Y., Bacqué M-H., F. GuérinPace (ed.). Le quartier.. Paris: La Découverte.

Hoyaux A-F. (2004). De l'espace domestique au monde domestiqué. In Collignon B., Staszak J.F. (dir). Espace domestiques. Paris : Éditions Bréal.

Hubert J.P. (1993). La Discontinuité critique. Essai sur les principes à priori de la géographie humaine. Paris: Publications de la Sorbonne.

Lazzarotti O. (2006). Habiter. La condition géographique. Paris: Belin.

Lussault M. (2007). L'homme spatial; la construction sociale de l'espace humain. Paris: Seuil.

Margier A. (2013). La cohabitation dans les espaces publics: conflits d'appropriation entre riverains et populations marginalisées à Montréal et Paris. Thèse de Doctorat en Études Urbaines, Université du Québec à Montréal.

Mitchell D. (2003). The right to the city. Social Justice and the Fight for Public Space. New-York: Guilford Press.

Moles A.A., Rohmer É. (1978). Psychologie de l'espace. Paris: Casterman.

Paugam S. (1991). La disqualification sociale.. Paris: Presses Universitaires de France.

Parazelli M. (1997). Pratiques de "socialisation marginalisée» et espace urbain: le cas des jeunes de la rue à Montréal (1985-1995). Thèse de doctorat en études urbaines, Université du Québec à Montréal.

Parazelli M. (2000). L'imaginaire familialiste et l'intervention sociale auprès des jeunes de la rue. Santé mentale au Québec, 2. doi: 10.7202/014451ar

Parazelli M. (2002). La rue attractive, parcours et pratiques identitaires des jeunes de la rue. Paris-Montreal: Presses de l'Université du Québec.

Ripoll F., Veschambre V. (2006). L'appropriation de l'espace: une problématique centrale pour la géographie sociale. In Séchet R., Veschambre V. Penser et faire la géographie sociale. Rennes: Presses Universitaires de Rennes.

Serfaty-Garzon Perla. (2003). Appropriation. In Segaud M., Brun J., Driant J.C. (Dir.). Dictionnaire de l'habitat et du logement. Paris: Armand Colin.

Toubon J.C., Messamah K. (1990). Centralité immigrée. Paris: L'Harmattan.

Sloterdijk P. (2005). Écumes, Sphères III. Paris: Éditions Pluriel.

Sloterdijk P. (2011). Globes. Sphères II. Paris: Éditions Pluriel.

Zeneidi D. (2008). C'est pas nous qui sommes à la rue, c'est la rue qui est à nous.. In Séchet R., Garat I., Zeneidi D. Rennes: Espaces en transactions. Rennes: Presses Universitaires de Rennes. 